\title{
Introduction to the special issue: The Tenth International Conference on Health Policy
}

\author{
Xiao-Hua Andrew Zhou • Donald Hedeker
}

Published online: 29 October 2014

(C) Springer Science+Business Media New York 2014

This special issue of Health Services and Outcomes Research Methodology contains papers, based on presentations at the tenth International Conference on Health Policy Statistics (ICHPS) held October 5-7, 2013 at The Palmer House Hilton Hotel in Chicago. This special issue contains 6 high quality papers, which reflects the very high standard of research presented at the conference.

Over 260 statisticians, methodologists, and health policy experts attended the tenth ICHPS, which was co-chaired by Xiao-Hua Andrew Zhou of the University of Washington and Donald Hedeker of the University of Illinois at Chicago.

The meeting's theme, "Statistically Engaged Health Care Policy," reflected the growing importance of the collaboration and engagement of statisticians in evaluating and improving health care policy in the US and throughout the world.

Major presentations were from a range of topics, including comparative effectiveness research, causal inference, longitudinal data, missing data, propensity score methods, data synthesis, personalized medicine and dynamic treatment regimes, and methods for patientreported outcomes.

Keynote addresses were given by Seth Eisen, Office of Research and Development of the Department of Veteran Affairs, on "The Reincarnation of Health Policy Statisticians," and Sharon-Lise Normand, Department of Health Care Policy at the Harvard Medical School, on "Statistics and Health Policy in 2013-Progresses and Retreats." Also, the Plenary address was delivered by Robert Gibbons, Departments of Medicine and Health

\footnotetext{
X.-H. A. Zhou $(\bowtie)$

Department of Biostatistics, U.S. Department of Veterans Affairs Seattle Medical Center, University of Washington, Seattle, USA

e-mail: azhou@uw.edu

D. Hedeker

Division of Epidemiology \& Biostatistics and Institute for Health Research \& Policy, School of Public Health, University of Illinois at Chicago, Chicago, USA

e-mail: hedeker@uic.edu
} 
Studies, and Director of the Center for Health Statistics at the University of Chicago, on "The Future of Mental Health Measurement."

Due to the federal government shutdown in October, a number of government workers could not travel and attend the conference. Indeed, Dr. Eisen, one of the keynote speakers, could not attend the conference in person, but instead delivered his talk over the phone, with the help of co-chair Andrew Zhou. Also, several presentations and one workshop had to be cancelled. This was indeed unfortunate, but did not detract in a major way from the quality of the conference.

The conference also included presentation of the Health Policy Statistics Section's biennial awards. Honored with the Long-Term Excellence Award were Naihua Duan, Professor Emeritus of Columbia University, and Robert Gibbons of the University of Chicago. A Mid-Career Award was presented to Susan Paddock of RAND Corporation. Fifteen students were recognized and received student travel awards for their presentations at the conference.

An entertainment event on Thursday evening included a performance by The Polkaholics, a band led by Don Hedeker, at Phyllis' Musical Inn in the Wicker Park neighborhood of Chicago. This performance was especially memorable as it featured Brad Carlin, of the University of Minnesota, joining the band on keyboards and vocals for many songs. As the band performed the hour-long set, several eminent statisticians were seen dancing to the crazy polka music!

The ten conference workshops (attracting nearly 400 attendees) included ones on Hierarchical Models for Longitudinal-Survival Data, Missing Data in Longitudinal Studies, Causal Inference, Propensity Score Methods, and Comparative Effectiveness Research. Co-chair Andrew Zhou concluded the conference by leading an informative workshop on Estimation of Re-Identification Risk in De-Identified Data.

The 11th ICHPS—-planned for year 2015-will be co-chaired by Kelly Zou of Pfizer, Inc., and Recai Yucel of SUNY Albany. For more information and details, contact Zou at Kelly.Zou@pfizer.com or Yucel at ryucel@uamail.albany.edu. 